\title{
Assessing the need and benefits of home tonometers in the management of patients with glaucoma
}

This article was published in the following Dove Press journal:

Clinical Optometry

24 July 2013

Number of times this article has been viewed

\section{Pinakin Gunvant Davey' \\ Kiana Nouri² \\ Samantha Zaczyk' \\ 'Western University of Health Sciences, College of Optometry, Pomona, CA, USA; ${ }^{2}$ Western University of Health Sciences, College of Osteopathic Medicine of the Pacific, Pomona CA, USA}

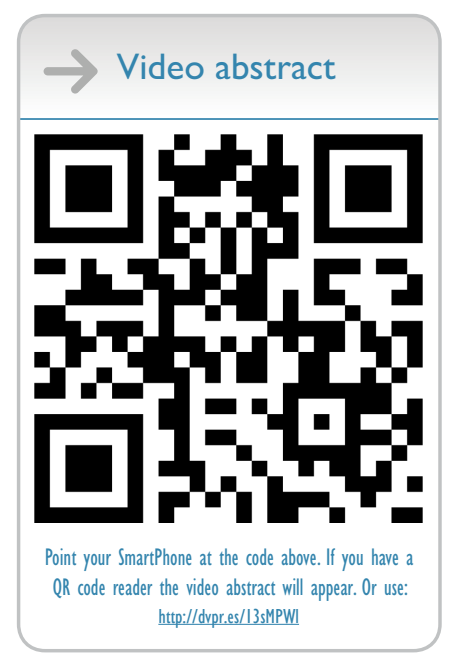

Correspondence: Pinakin Gunvant Davey Western University of Health Sciences, College of Optometry, 309 East Second Street, Pomona, CA 91766, USA

Tel +l 9094698473

Email contact@pinakin-gunvant.com

\begin{abstract}
Intraocular pressure (IOP) measurement remains an integral part of managing patients with or at risk for glaucoma. The current gold standard, the Goldmann applanation tonometer, is influenced by ocular parameters, both extrinsic and intrinsic, that can lead to long- and short-term fluctuations and measurement errors. The biggest of all limitations of Goldmann-type tonometers is that the device provides a cross-sectional picture of IOP levels. To get an overall picture, including nocturnal IOP estimates, a home tonometry unit can provide valuable information. This article discusses the various sources that influence IOP measurement, home tonometers that are currently available, and the growing body of evidence that shows how home tonometry can be helpful in the care of individuals at risk for progression of glaucoma.
\end{abstract}

Keywords: home tonometry, intraocular pressure, sources of errors, telemetry

\section{Introduction}

Primary open-angle glaucoma is a slow, progressive, optic neuropathy with a characteristic optic disc excavation, progressive retinal nerve fiber layer loss, and acquired visual field loss. The exact pathophysiology of primary open-angle glaucoma is yet to be fully established, and a definite biological marker is not currently available. In the absence of this knowledge, various population-based studies ${ }^{1-8}$ and clinical investigations have shed light on risk factors that predispose individuals to the disease. Intraocular pressure (IOP) is arguably the most important of the risk factors, as it remains the only modifiable risk factor that helps manage and control disease progression. With such importance placed on measured IOP, it becomes vital to measure this variable as accurately and frequently as possible. Thus, accurate measurements are required both in the office and at home. This article highlights the importance of IOP and physiological parameters, which can cause true and erroneous short- and long-term fluctuations in measured IOP values, thus making a case for the need of home tonometry. This article reviews various home tonometry devices and the evidence of its benefits in managing individuals at risk for glaucoma.

\section{Importance of IOP in glaucoma management}

Although IOP levels cannot fully explain glaucomatous pathology, its importance in managing patients with glaucoma should not be underestimated. Of the various risk factors known, such as age, race, family history, central corneal thickness, ocular perfusion, diabetes, and genetics, IOP is the only modifiable risk factor. Numerous population-based studies ${ }^{1-8}$ have shown increased prevalence of primary open-angle glaucoma with an increase in IOP. In addition, numerous randomized controlled 
clinical trials have shown that reduction in IOP lessens the risk for visual field progression. ${ }^{9-14}$ These population-based studies and treatment trials cumulatively provide evidence that IOP plays an important role in pathogenesis of glaucoma. It must be remembered that having a cutoff level of IOP that is $21 \mathrm{mmHg}$ or greater is an arbitrary cutoff and may yield low specificity as a diagnostic criterion. As low as $13 \%,{ }^{15}$ or as high as $71 \%,{ }^{16}$ of individuals having IOP values of $21 \mathrm{mmHg}$ or greater, may signify glaucoma.

\section{Factors influencing measurement of IOP}

As clinicians, we look for data helpful in the management of disease, and we prefer data that are consistent, reproducible, accurate, and that do not greatly vary. IOP values, similar to many other physiological measurements, are in a state of flux and can vary both short- and long-term. Furthermore, to complicate the issue, there are numerous ocular biomechanical factors such as central corneal thickness, corneal curvature, corneal rigidity, and hydration that can lead to errors in clinical IOP measurement. ${ }^{17}$

There are numerous factors that lead to frank errors in IOP measurements, and there are other sources that lead to fluctuation in IOP levels. ${ }^{17}$ Since most current IOP measurement techniques only give an estimate at a given moment in time, short-term fluctuations can lead to a significant overestimation or underestimation of IOP from its true levels.

There are several factors that can lead to short- or long-term fluctuations of IOP. Overall IOP is thought to show polygenic inheritance, with a definite environmental contribution. ${ }^{18}$ The factors that can contribute to long-term fluctuations or variations in IOP are age, blood pressure, and seasonal variations. ${ }^{19-21}$ Although these factors tend to cause long-term fluctuations in IOP, its clinical importance is minimal. These factors are also very difficult to tease apart from those that induce short-term variations in IOP.

The factors that contribute to short-term variations in IOP values are diurnal variations, body posture, exercise, eye movements, activities causing Valsalva-like maneuver, and food and drug effects. These factors can pose a significant problem in clinical management, as IOP level is one of the integral measurements that helps decide the clinical efficacy of glaucoma medications and, to some extent, the management strategy in patients with or at risk for glaucoma. The diurnal variations in IOP levels are well known to occur in ocular-normal individuals. These IOP variations are to some extent exaggerated in patients with open-angle glaucoma. ${ }^{22-26}$ Although interindividual variations may be significant, on average IOP levels are highest before awakening and in the early morning hours, and are minimal late at night. ${ }^{27}$ This theory has been questioned recently, however: IOP may in fact increase at night, ${ }^{28-30}$ which may in part be responsible for progressive glaucomatous damage. ${ }^{30}$ Most of the variations in IOP that occur in the diurnal cycle are attributable to aqueous humor production, which varies as a function of levels of circulating cortisol and catecholeamines. ${ }^{31,32}$ Changes in aqueous humor inflow may not fully explain the diurnal fluctuations of IOP, ${ }^{30,33}$ and perhaps episcleral venous pressure variations contribute to the effect. ${ }^{30}$

The diurnal variation of IOP can be in the range of $3-9 \mathrm{mmHg}$, and obtaining this value may be fundamental to establishing a good estimate of target IOP range. In addition, diurnal variations become important in the longterm care of patients and in the follow-up of patients with glaucoma. Thus, the IOP measurement taken in the office becomes an important variable to be considered, particularly in follow-up visits related to IOP checks and when evaluating the efficacy of IOP-lowering medications in patients. Diurnal IOP measurement is very difficult to perform, particularly with clinical devices, and may require hospitalization. The availability of home tonometers will benefit in obtaining this difficult measurement.

Postural variations in IOP become significant because of measurement-related issues in clinics and because of lifestyle issues of the patients. Changes in body position from erect to supine or from erect to upside down can lead to changes in IOP on the magnitude of $16 \mathrm{mmHg}$ and $30 \mathrm{mmHg}$, respectively. ${ }^{34}$ This short-term increase in IOP is a result of changes in the central venous pressure, which consequently produces changes in episcleral venous pressure. ${ }^{34}$ With the increase in popularity of yoga as a complementary and alternative medicinal technique, ${ }^{35,36}$ it is a fair question to ask whether these exercise postures, some of which require complete inversion, pose a risk to ocular health. In an observational prospective cohort, Baskaran et $\mathrm{al}^{36}$ show that assuming a complete inverted position leads to an immediate twofold increase in IOP from baseline that both persisted for the duration of the event and returned immediately to normal levels with assuming a seated position. This effect was seen in both Asian Indians and Caucasians.

Exercise has a variable effect on IOP: aerobic exercise causes lowering of IOP, ${ }^{37-40}$ whereas isometric exercises such as lifting weights may produce a small IOP increase during exertion. ${ }^{41}$ Such changes in IOP are not damaging in healthy adults $^{36}$ but may play a role in open-angle glaucoma, ${ }^{42}$ particularly in progressive damage in patients with open-angle 
glaucoma ${ }^{43}$ and angle-closure glaucoma, ${ }^{44}$ as postulated previously. Thus, caution is warranted among yoga-practicing patients with glaucoma. In addition, unilateral forced nostril breathing (UFNB), which occurs across a wide range of exertion from exercise, makes very specific changes to IOP because of its different effects on the autonomic nervous system. Right UFNB stimulates sympathetic activity by way of left-hemispheric stimulation, whereas left UFNB stimulates parasympathetic activity by way of right-hemispheric stimulation. ${ }^{45}$ As a result, IOP decreases with right nostril breathing and increases with left nostril breathing. Chen et a ${ }^{46}$ showed that right nostril breathing decreased IOP in right and left eyes significantly ( $9.6 \%$ and $6.7 \%$, respectively), whereas left nostril breathing increased IOP an average of $5.7 \%$ and $2.5 \%$, which was not statistically significant.

The increase in IOP during isometric exercise is a result of a Valsalva-like maneuver, which is an expiratory effort with the glottis closed. ${ }^{47}$ Proper breathing technique during exercises that involve lifting heavy weights is important to avoid elevation in IOP. Similarly, a lesser-known effect is a result of attempted inhalation against a closed glottis, called the Mueller maneuver, which causes a decrease in IOP. ${ }^{48}$ Both Valsalva and Mueller maneuvers have clinical significance, especially in nervous or anxious individuals who may hold their breath while IOP is being measured ${ }^{47}$ The effect on IOP of holding one's breath is further exaggerated by the strain produced while positioned in a slit lamp for measuring IOP, particularly in heavyset individuals.

Similarly, when positioned at the slit lamp, voluntary widening ${ }^{49}$ or attempted or involuntary narrowing of the palpebral fissure ${ }^{50}$ can produce a measurable transient spike in IOP. Prior work by Bain and Maurice in $1959^{51}$ showed that if the venous flow is restricted by constriction around the neck, using a sphygmomanometer cuff to $40 \mathrm{mmHg}$ leads to a doubling of IOP from baseline. It was postulated that restricting the venous circulation, particularly in the jugular vein, results in elevated episcleral venous pressure, which subsequently leads to elevated IOP. ${ }^{50,51}$ The same scenario is simulated to a lesser extent when wearing tight clothing, such as a tight necktie, which is shown to elevate IOP in both ocular healthy patients and patients with glaucoma. ${ }^{52}$ However, some other researchers have failed to confirm such an effect in ocular healthy individuals..$^{53,54}$ The discordance in the outcome of these studies suggests that a more complex mechanism is involved in IOP changes resulting from tight clothing. Theelen et $\mathrm{al}^{54}$ found that the elevation in IOP resulting from tight clothing may be an artifact, and in reality, positioning of the patient in slit lamp is truly responsible for the elevation of IOP. In addition, it may be possible that the elevated venous pressure may have a difference in the amount of elevation in IOP in patients with glaucoma when compared with ocular healthy individuals.

Examining the normal physiological processes, we find that the measured IOP varies continuously with both cardiac and respiratory pulses and is seen in the pulsating mires when one performs Goldmann applanation tonometry. This is of limited importance in Goldmann applanation tonometry, as the mires are aligned midpoint of pulsations; ${ }^{55}$ furthermore, the time taken to perform tonometry is greater than the cyclic periodicity of the cardiac and respiratory pulse. However, this is of particular relevance to noncontact tonometry, as the measurements are obtained near-instantaneously. ${ }^{56}$ Thus, obtaining multiple measurements and averaging IOP is important when obtaining IOP using noncontact tonometers. ${ }^{57}$

There are numerous instances when multiple measurements of IOP are needed. This may be in a nervous or uncooperative individual in whom the first measurement failed to yield reliable values, or it may be just to recheck measurements obtained. Repeated applanation using Goldmann-type tonometers is known to decrease IOP in the ipsilateral eye, ${ }^{57-61}$ as well as in the contralateral eye to a lesser extent. ${ }^{60,62,63}$ The decrease in IOP resulting from repeated applanation is a complex process, and neither tonographic effect nor changes in corneal biomechanics resulting from repeated applanation can fully explain the drop in IOP. ${ }^{17,61,63}$ The process of repeated applanation is not an issue when measurements are performed near instantaneously, as in noncontact tonometry, but it may be a problem if a time interval of a few minutes passes between repeated measurements using Goldmann-type tonometers ${ }^{57-61}$ and noncontact tonometry. ${ }^{64}$ It would be ideal to allow a 10-minute time interval if the measurements are to be repeated using Goldmann-type tonometers.

Other factors that lead to fluctuations in IOP measurement are related to the consumption of certain foods, beverages, and recreational drugs. ${ }^{65-78}$ Consumption of large quantities of water (500-1000 mL) has been shown to increase IOP in humans. ${ }^{65-69}$ Prior studies had proposed that a waterdrinking test could be used as a predictive test to diagnose glaucoma ${ }^{65,66}$ However, more recently, the water-drinking test has been used to identify peak increases of IOP. ${ }^{67-69}$ In healthy individuals, peak increases of an average of $2.24 \mathrm{mmHg}$ occurred 10 minutes after water-loading. ${ }^{69}$ IOP increases on average of $5.00 \mathrm{mmHg}$ were demonstrated in patients with glaucoma, with choroidal expansion also being associated with an increase in IOP, particularly in patients with angle- 
closure glaucoma. ${ }^{72}$ Caffeine $\mathrm{C}^{37,70}$ and excessive consumption of water can cause a transient increase in IOP, 37,71 whereas consumption of alcohol leads to a transient decrease in IOP ${ }^{37,57,73}$ Smoking tobacco in the form of cigarettes can lead to a transient increase in IOP, ${ }^{74}$ whereas smoking marijuana can lead to a decrease in IOP. ${ }^{75-78}$ Clinicians will have to pay particular attention to patients when IOP values do not match other clinical findings.

Ocular biometric factors such as myopia and accommodation can also cause fluctuations in IOP. A mean decrease of $1.8 \mathrm{mmHg}$ was noted after an accommodation task in both young progressing myopes and emmetropes. ${ }^{79}$ These short-term variations in IOP make the case for having numerous IOP measurements and, possibly, measuring at different times of the day before initiating therapy or making changes to the management of patients. This may not be always possible in clinical setting or during office visits, and thus home tonometry becomes crucial in the management of glaucoma.

\section{Home tonometry}

Home tonometry as a concept has not picked up momentum because older attempts required topical anesthetics ${ }^{80,81}$ and involved expensive technology. In recent years, attempts have been made to make the device more affordable and not require anesthesia. Measuring IOP frequently and diurnally affords numerous advantages: home tonometry may give a truer picture of IOP values; measurements will not be influenced by errors induced in clinical settings, such as slit lamp position; and it may provide a decreased level of anxiety, particularly in nervous patients. On a more basic science level, measuring IOP around the clock provides better understanding of the aqueous humor dynamics, pathogenesis in glaucoma, and better understanding of the efficacy of IOP-lowering medications. Clinically, measuring the diurnal curve of IOP gives an idea of the fluctuations and range of IOP that can be used to better judge the risk level of patients and appropriately set up the target IOP range. Knowledge of diurnal IOP values may also be of great use in tailoring management in patients; that is, prescribing the appropriate medications when they are needed the most. It may help in the diagnosis and management of normal tension glaucoma and other patients with progressing glaucoma or suspect cases. In addition, high-risk cases such as pediatric glaucoma, where there is a need to closely monitor the efficacy of treatment, provide clinicians an insight that is not available by measurements obtained during an office visit.

\section{Proview home tonometer}

The Proview pressure phosphene tonometer developed by Fresco $^{82}$ provided IOP measurements through the eyelids, did not require anesthesia, and was a fraction of the price of previous home tonometers. However, the measurements obtained by the Proview pressure phosphene tonometry were variable, and its agreement with the Goldmann applanation tonometer was moderate at best. ${ }^{82-88}$ Similar to other clinical tonometers, the Proview pressure phosphene tonometer only gives a snapshot of IOP in time.

\section{Icare rebound tonometer}

The Icare rebound tonometer (Icare; Helsinki, Finland) is a device that can measure IOP estimates without the use of topical anesthesia. It consists of an assembly of two coils coaxial to a probe shaft that bounce a magnetized probe off the cornea and detect the deceleration of the probe caused by the eye. A moving magnet within a coil induces changes in the voltage that are detected by the tonometer sensor. The voltage produced is proportional to the probe speed, and the deceleration speed seems to correlate best with IOP. ${ }^{89}$ The probe tip has a 1-mm-diameter plastic cover to minimize corneal damage. The probe tip is disposable between patients, so disinfection is not necessary.

When the device is activated for measurement, a spring drives the wire and probe forward rapidly. When the probe hits the cornea, it decelerates; the deceleration is more rapid if the IOP is high and slower if the IOP is low. The speed of deceleration is measured internally, and a chip calculates the IOP. As the contact with the cornea is momentary, no anesthetic is necessary. ${ }^{90}$

This device is now modified for use as a home tonometer and is called Icare ONE. The ability of the Icare ONE to provide IOP estimates as a home tonometer has been evaluated in a few recent studies. ${ }^{91-93}$ Although it is known that rebound tonometry may be influenced by corneal parameter, ${ }^{94}$ its smaller design and the fact that it has no requirement for anesthetic before measurements makes it an ideal instrument for at-home and repeated use.

Studies have shown that the Icare ONE has a good agreement with the Goldmann applanation tonometer in about $70 \%$ of cases. ${ }^{91,92}$ In addition, large fluctuations in IOP have been identified as an independent risk factor in glaucoma. ${ }^{95}$ Two recent studies have evaluated the use of Icare in pediatric population: Gandhi et $\mathrm{al}^{91}$ compared Icare ONE with the clinical gold standard in the pediatric population; good concordance in two-thirds of cases was found, and it was determined that the device was easy to understand and use 
by the caregiver. Flemmons et $\mathrm{al}^{92}$ measured IOP diurnally, using Icare ONE, and concluded that about $30 \%$ of patients had a peak IOP measurement of $6 \mathrm{mmHg}$ greater when measured diurnally versus at office measurement. ${ }^{92}$ Although a similar study with Icare ONE has not been performed in the adult population, one can hypothesize that such variations may exist in adults with primary open-angle glaucoma, and the measurements will be of great value in managing such patients.

Because the rebound tonometer has extremely short contact time with the eye, one may get a reading at any point in the IOP pulse cycle, and thus multiple readings need to be averaged to obtain a clinically useful value.

\section{Continuous monitoring of IOP}

All tonometers, both in the office and in the home, provide a snapshot of aqueous humor dynamics and IOP in time. The need for 24-hour continuous IOP measurement and types of devices are examined in detail in a separate review article. ${ }^{96}$ It would be ideal if IOP were measured automatically by a sensor inside or around the eye. This concept is not new: more than 40 years ago, Collins proposed that an encapsulated parallel capacitive circuit, when placed in the anterior chamber or in the posterior chamber, can measure IOP continuously and will not require an additional external power source. ${ }^{97}$ More recently, work from Walter and colleagues has led to the development of an intraocular lens that was capable of measuring IOP continuously. ${ }^{98,99}$ Chen et al ${ }^{100}$ have successfully shown both in vitro and in vivo testing results of an implantable unpowered parylene-based micro-electro-mechanicalsystems. ${ }^{100}$ The parylene-based sensor has been shown to be biocompatible, with minimally invasive implantation and no postoperative complications. This represents one of the most advanced techniques in wireless and continuous IOP monitoring devices. Fully implantable telemetry systems that allow continuous wireless monitoring of IOP were first tested in rabbits and rodents before their successful implantation in nonhuman primates. ${ }^{101}$ Although theoretically these permanent devices that are implanted inside the eye may give better IOP measurements that are less influenced by ocular biomechanical factors, the implantation of these permanent devices requires surgical procedures, and their long-term safety and biocompatibility are always a concern. A device that can be removed and that can be used on an ad hoc basis may be a safer alternative.

Prior attempts to create contact lens tonometers with built-in pressure sensors were limited because of the types of contact lens materials available. Rigid lenses were used, which were far from comfortable, ${ }^{102-104}$ and the data obtained were also not very reliable. In addition, the values obtained were influenced by ocular biomechanics, which caused variations in IOP measurement. ${ }^{103,104}$ These types of devices would have a vital role to play not only in 24-hour diurnal measurement of IOP but also in measuring ultra-short-term fluctuations in IOP that occur as a result of physiological changes such as saccadic eye movement. ${ }^{105}$

More recently, Sensimed (Lausanne, Switzerland) has developed Triggerfish ${ }^{\circledR},{ }^{106}$ a disposable silicone contact lens unit that is capable of measuring pressure variation in arbitrary units continuously. This was developed by Leonardi et al ${ }^{107,108}$ and uses a soft contact lens with a strain gauge that allows the measurement of change in curvature of the cornea. A telemetry microprocessor and an antenna are also embedded into the contact lens for wireless power and data transfer. The principle of pressure measurement by Triggerfish $^{\circledR}$ is based on the fact that prior studies have shown that a change of $1 \mathrm{mmHg}$ in IOP typically produces a change of $3 \mu \mathrm{m}$ in corneal curvature in a cornea with a $7.8-\mathrm{mm}$ radius of curvature. ${ }^{109,110}$ The pressure variation is measured every 5-10 minutes for a period of 30-60 seconds, giving a total of up to 144 measurements during a period of 24 hours. The Triggerfish ${ }^{\circledR}$ is available in 3 base curves (9.0, 8.7, and $8.4 \mathrm{~mm}) .{ }^{111,112}$ The complete unit consists of a soft patch, worn around the eye, that allows an unobstructed view and that telemetrically receives data from contact lenses. A wire from the patch subsequently transmits data to a recorder attached to the patient's clothes. The device is approved for use in Europe and costs about $€ 500$ (approximately US\$700), and is not available for sale in the United States. The published literature on clinical efficacy of Triggerfish ${ }^{\circledR}$ is increasing. Freiberg et $\mathrm{al}^{113}$ showed that there was a significant change in central corneal thickness $(2.7 \%)$ after overnight wear of contact lens for IOP measurement. This change in corneal thickness was expected because of the hypoxic environment during eye closure. However, this change in corneal thickness was not significantly correlated to the IOP signal change obtained by the device. ${ }^{113}$

The reproducibility of pressure patterns as measured by the contact lenses units was evaluated by Mansouri et al $^{112}$ in 37 patients who had a confirmed or suspected diagnosis of glaucoma. ${ }^{112}$ The researchers found that these units, which were worn for a 24-hour period twice (1 week apart), showed a fair-to-good reproducibility. That is, the correlation coefficient (Pearson correlation $r$ ) of the values generated by the device during the two sessions was 0.59 overall. The individuals not receiving glaucoma medications showed more variability 
(Pearson correlation $r, 0.51$ ) compared with individuals who were receiving glaucoma medications (Pearson correlation $r$, 0.63). The authors concluded that such a device may play a role in management of patients with glaucoma.

Mansouri and Shaarawy ${ }^{111}$ evaluated the contact lens pressure-measuring device in 15 patients with progressive damage resulting from open-angle glaucoma, despite controlled IOPs measured during office hours. They found that $69 \%$ of those patients had the highest IOP recorded during nocturnal periods, with prolonged peaks of pressure spikes greater than 1 hour seen in $80 \%$ of patients outside normal office hours. Obtaining the information of pressure fluctuations using the contact lens pressure measuring-device led to a change in management strategy in $73 \%$ of patients involved in the study.

The safety of this type of device was evaluated by two groups. ${ }^{112,113}$ As expected, the adverse effects for the overnight wear of these contact lens-based IOP measuring units were superficial punctuate keratitis and conjunctival hyperemia, which required no more than palliative treatment. Although blurred vision was reported by patients, the best corrected visual acuity remained unchanged before and after overnight wear of contact lenses. ${ }^{112}$

Although excellent in its concept and for providing useful data, the measurements obtained using contact lens devices have significant room for improvement. There will be a transition phase before the arbitrary units that are generated by the devices are understood in terms of IOP that is measured and used in managing patients. The arbitrary units output by this device are affected by blinks and eye movement, both of which can be filtered mathematically, as these produce characteristic short spikes in measurement. The other factors that may affect measurement of these contact lens devices are change in stromal hydration throughout the day and night, corneal biomechanics, keratometry, corneal diameter, axial length, or scleral thickness and rigidity; these warrant further investigation. $^{96}$

Home tonometry is at an interesting point or phase of development, as prior units required anesthetic to measure IOP and were extremely expensive. The newer-generation devices do not require anesthetic, which is very welcome. With the advances in telemetric units, both implantable units and disposable noninvasive contact techniques, we are perhaps at the breaking point of obtaining information on aqueous humor dynamics that was not available previously. More research performed on the safety, validity, and clinical applicability of these devices will lead to establishing new clinical protocols on how to use them appropriately in clinical care.

\section{Disclosure}

The authors report no conflicts of interest in this work.

\section{References}

1. Dielemans I, Vingerling JR, Wolfs RC, Hofman A, Grobbee DE, de Jong PT. The prevalence of primary open-angle glaucoma in a population-based study in The Netherlands. The Rotterdam Study. Ophthalmology. 1994;101(11):1851-1855.

2. Sommer A, Tielsch JM, Katz J, et al. Relationship between intraocular pressure and primary open angle glaucoma among white and black Americans. The Baltimore Eye Survey. Arch Ophthalmol. 1991;109(8):1090-1095.

3. Mitchell P, Smith W, Attebo K, Healey PR. Prevalence of open-angle glaucoma in Australia. The Blue Mountains Eye Study. Ophthalmology. 1996;103(10):1661-1669

4. Leske MC, Connell AM, Schachat AP, Hyman L. The Barbados Eye Study. Prevalence of open angle glaucoma. Arch Ophthalmol. 1994;112(6):821-829.

5. Quigley HA, West SK, Rodriguez J, Munoz B, Klein R, Snyder R. The prevalence of glaucoma in a population-based study of Hispanic subjects: Proyecto VER. Arch Ophthalmol. 2001;119(12):1819-1826.

6. Leibowitz HM, Krueger DE, Maunder LR, et al. The Framingham Eye Study monograph: An ophthalmological and epidemiological study of cataract, glaucoma, diabetic retinopathy, macular degeneration, and visual acuity in a general population of 2631 adults, 1973-1975. Surv Ophthalmol. 1980;24(Suppl):335-610.

7. Klein BE, Klein R, Sponsel WE, et al. Prevalence of glaucoma. The Beaver Dam Eye Study. Ophthalmology. 1992;99(10):1499-1504.

8. Weih LM, Nanjan M, McCarty CA, Taylor HR. Prevalence and predictors of open-angle glaucoma: results from the visual impairment project. Ophthalmology. 2001;108(11):1966-1972.

9. Bonomi L, Marchini G, Marraffa M, et al. Prevalence of glaucoma and intraocular pressure distribution in a defined population. The Egna-Neumarkt Study. Ophthalmology. 1998;105(2):209-215.

10. Leske MC, Connell AM, Wu SY, Hyman LG, Schachat AP. Risk factors for open-angle glaucoma. The Barbados Eye Study. Arch Ophthalmol. 1995;113(7):918-924.

11. Comparison of glaucomatous progression between untreated patients with normal-tension glaucoma and patients with therapeutically reduced intraocular pressures. Collaborative Normal-Tension Glaucoma Study Group. Am J Ophthalmol. 1998;126(4):487-497.

12. The effectiveness of intraocular pressure reduction in the treatment of normal-tension glaucoma. Collaborative Normal-Tension Glaucoma Study Group. Am J Ophthalmol. 1998;126(4):498-505.

13. Gordon MO, Beiser JA, Brandt JD, et al. The Ocular Hypertension Treatment Study: baseline factors that predict the onset of primary open-angle glaucoma. Arch Ophthalmol. 2002;120(6):714-720; discussion 829-830.

14. Leske MC, Heijl A, Hussein M, Bengtsson B, Hyman L, Komaroff E; Early Manifest Glaucoma Trial Group. Factors for glaucoma progression and the effect of treatment: the early manifest glaucoma trial. Arch Ophthalmol. 2003;121(1):48-56.

15. Heijl A, Leske MC, Bengtsson B, Hyman L, Bengtsson B, Hussein M; Early Manifest Glaucoma Trial Group. Reduction of intraocular pressure and glaucoma progression: results from the Early Manifest Glaucoma Trial. Arch Ophthalmol. 2002;120(10):1268-1279.

16. The Advanced Glaucoma Intervention Study (AGIS): 7. The relationship between control of intraocular pressure and visual field deterioration. The AGIS Investigators. Am J Ophthalmol. 2000;130(4): 429-440.

17. Whitacre MM, Stein R. Sources of error with use of Goldmann-type tonometers. Surv Ophthalmol. 1993;38(1):1-30.

18. Carbonaro F, Andrew T, Mackey DA, Spector TD, Hammond CJ. Heritability of intraocular pressure: a classical twin study. $\mathrm{Br} J$ Ophthalmol. 2008;92(8):1125-1128. 
19. Bengtsson B. Some factors affecting the distribution of intraocular pressures in a population. Acta Ophthalmol (Copenh). 1972;50(1):33-46.

20. Carel RS, Korczyn AD, Rock M, Goya I. Association between ocular pressure and certain health parameters. Ophthalmology. 1984;91(4): 311-314.

21. Shiose Y. The aging effect on intraocular pressure in an apparently normal population. Arch Ophthalmol. 1984;102(6):883-887.

22. Henkind P, Leitman M, Weitzman E. The diurnal curve in man: new observations. Invest Ophthalmol. 1973;12(9):705-707.

23. Drance SM. The significance of the diurnal tension variations in normal and glaucomatous eyes. Arch Ophthalmol. 1960;64:494-501.

24. Liu JH. Diurnal measurement of intraocular pressure. J Glaucoma. 2001;10(5 Suppl 1):S39-S41.

25. Liu JH, Zhang X, Kripke DF, Weinreb RN. Twenty-four-hour intraocular pressure pattern associated with early glaucomatous changes. Invest Ophthalmol Vis Sci. 2003;44(4):1586-1590.

26. Sehi M, Flanagan JG, Zeng L, Cook RJ, Trope GE. Relative change in diurnal mean ocular perfusion pressure: a risk factor for the diagnosis of primary open-angle glaucoma. Invest Ophthalmol Vis Sci. 2005;46(2):561-567.

27. Wan XL, Sears J, Chen S, Sears M. Circadian aqueous flow mediated by beta-arrestin induced homologous desensitization. Exp Eye Res. 1997;64(6):1005-1011.

28. Liu JH, Kripke DF, Hoffman RE, et al. Elevation of human intraocular pressure at night under moderate illumination. Invest Ophthalmol Vis Sci. 1999;40(10):2439-2442.

29. Liu JH, Kripke DF, Twa MD, et al. Twenty-four-hour pattern of intraocular pressure in the aging population. Invest Ophthalmol Vis Sci. 1999;40(12):2912-2917.

30. Sit AJ, Nau CB, McLaren JW, Johnson DH, Hodge D. Circadian variation of aqueous dynamics in young healthy adults. Invest Ophthalmol Vis Sci. 2008;49(4):1473-1479.

31. Brubaker RF. The physiology of aqueous humor formation. In: Drance S, Neufeld A, editors. Applied Pharmacology in the Medical Treatment of Glaucoma. Orlando: Grune and Stratton; 1984.

32. Reiss GR, Lee DA, Topper JE, Brubaker RF. Aqueous humor flow during sleep. Invest Ophthalmol Vis Sci. 1984;25(6):776-778.

33. Brubaker RF. Flow of aqueous humor in humans [The Friedenwald Lecture]. Invest Ophthalmol Vis Sci. 1991;32(13):3145-3166.

34. Tarkkanen A, Leikola J. Postural variations of the intraocular pressure as measured with the Mackay-Marg tonometer. Acta Ophthalmol (Copenh). 1967;45(4):569-575.

35. Fisher P, Ward A. Complementary medicine in Europe. BMJ. 1994; 309(6947):107-111.

36. Baskaran M, Raman K, Ramani KK, Roy J, Vijaya L, Badrinath SS. Intraocular pressure changes and ocular biometry during Sirsasana (headstand posture) in yoga practitioners. Ophthalmology. 2006;113(8): 1327-1332.

37. Buckingham T, Young R. The rise and fall of intra-ocular pressure: the influence of physiological factors. Ophthalmic Physiol Opt. 1986;6(1): 95-99.

38. Marcus DF, Krupin T, Podos SM, Becker B. The effect of exercise on intraocular pressure. I. Human beings. Invest Ophthalmol. 1970;9(10) 749-752.

39. McDaniel DR, Tribbey CL, Tobias GS. Effects of moderate exercise on intraocular pressure. Am J Optom Physiol Opt. 1983;60(3):154-157.

40. Passo MS, Goldberg L, Elliot DL, Van Buskirk EM. Exercise training reduces intraocular pressure among subjects suspected of having glaucoma. Arch Ophthalmol. 1991;109(8):1096-1098.

41. Vieira GM, Oliveira HB, de Andrade DT, Bottaro M, Ritch R. Intraocular pressure variation during weight lifting. Arch Ophthalmol. 2006;124(9):1251-1254.

42. Rice R, Allen RC. Yoga in glaucoma. Am J Ophthalmol. 1985 15;100(5): 738-739.

43. Gallardo MJ, Aggarwal N, Cavanagh HD, Whitson JT. Progression of glaucoma associated with the Sirsasana (headstand) yoga posture. $A d v$ Ther. 2006;23(6):921-925.
44. Fahmy JA, Fledelius H. Yoga-induced attacks of acute glaucoma. A case report. Acta Ophthalmol (Copenh). 1973;51(1):80-84.

45. Werntz DA, Bickford RG, Shannahoff-Khalsa D. Selective hemispheric stimulation by unilateral forced nostril breathing. Hum Neurobiol. 1987;6(3):165-171.

46. Chen JC, Brown B, Schmid KL. Effect of unilateral forced nostril breathing on tonic accommodation and intraocular pressure. Clin Auton Res. 2004;14(6):396-400.

47. Aykan U, Erdurmus M, Yilmaz B, Bilge AH. Intraocular pressure and ocular pulse amplitude variations during the Valsalva maneuver. Graefes Arch Clin Exp Ophthalmol. 2010;248(8):1183-1186.

48. Lundmark PO, Trope GE, Flanagan JG. The effect of simulated obstructive apnoea on intraocular pressure and pulsatile ocular blood flow in healthy young adults. Br J Ophthalmol. 2003;87(11):1363-1369.

49. Moses RA, Carniglia PE, Grodzki WJ Jr, Moses J. Proptosis and increase of intraocular pressure in voluntary lid fissure widening. Invest Ophthalmol Vis Sci. 1984;25(8):989-992.

50. Jamal KN, Gürses-Ozden R, Liebmann JM, Ritch R. Attempted eyelid closure affects intraocular pressure measurement in open-angle glaucoma patients. Am J Ophthalmol. 2002;134(2):186-189.

51. Bain WE, Maurice DM. Physiological variations in the intraocular pressure. Trans Ophthalmol Soc UK. 1959;79:249-260.

52. Teng C, Gurses-Ozden R, Liebmann JM, Tello C, Ritch R. Effect of a tight necktie on intraocular pressure. Br J Ophthalmol. 2003;87(8):946-948.

53. Talty $\mathrm{P}, \mathrm{O}$ 'Brien PD. Does extended wear of a tight necktie cause raised intraocular pressure? J Glaucoma. 2005;14(6):508-510.

54. Theelen T, Meulendijks CF, Geurts DE, van Leeuwen A, Voet NB, Deutman AF. Impact factors on intraocular pressure measurements in healthy subjects. Br J Ophthalmol. 2004;88(12):1510-1511.

55. Kass MA. Standardizing the measurement of intraocular pressure for clinical research. Guidelines from the Eye Care Technology Forum. Ophthalmology. 1996;103(1):183-185.

56. Grolman B. A new tonometer system. Am J Optom Arch Am Acad Optom. 1972;49(8):646-660.

57. Moses RA, Arnzen RJ. Instantaneous tonometry. Arch Ophthalmol. 1983;101(2):249-252.

58. Moses RA. Repeated applanation tonometry. Ophthalmologica. $1961 ; 142: 663-668$.

59. Recep OF, Hasiripi H, Vayisoglu E, Kalayci D, Sarikatipoglu H. Accurate time interval in repeated tonometry. Acta Ophthalmol Scand. 1998;76(5):603-605.

60. Wilke K. Effects of repeated tonometry: genuine and sham measurements. Acta Ophthalmol (Copenh). 1972;50(4):574-582.

61. Gunvant P, Watkins RJ, Broadway DC, O'Leary DJ. Repeatability and effects of sequential measurements with POBF tonograph. Optom Vis Sci. 2004;81(10):794-799.

62. Krakau CE, Wilke K. On repeated tonometry. Acta Ophthalmol (Copenh). 1971;49(4):611-614.

63. Stocker FW. On changes in intraocular pressure after application of the tonometer; in the same eye and in the other eye. Am J Ophthalmol. 1958;45(2):192-196.

64. Gunvant P, Cohler M. Effect of repeated applanation on corneal biomechanical parameters and intraocular pressure measurements. Optom Vis Sci. 2010;87.

65. Schmidt K. Untersuchungen über Kapillarendothelstörungen bei Glaukoma simplex. [Studies on cappilary endothelium in chronic simple glaucoma]. Arch Augenheilkd. 1928;98:569-581. German.

66. Leydhecker W. The water-drinking test. Br J Ophthalmol. 1950;34(8):457-479.

67. Susanna R Jr, Vessani RM, Sakata L, Zacarias LC, Hatanaka M. The relation between intraocular pressure peak in the water drinking test and visual field progression in glaucoma. Br J Ophthalmol. 2005;89(10): 1298-1301.

68. Hatanaka M, Alencar LM, De Moraes CG, Susanna R Jr. Reproducibility of intraocular pressure peak and fluctuation of the water-drinking test. Clin Experiment Ophthalmol. 2013;41(4):355-359.

69. Read SA, Collins MJ. Water drinking influences eye length and IOP in young healthy subjects. Exp Eye Res. 2010;91(2):180-185. 
70. Higginbotham EJ, Kilimanjaro HA, Wilensky JT, Batenhorst RL, Hermann D. The effect of caffeine on intraocular pressure in glaucoma patients. Ophthalmology. 1989;96(5):624-626.

71. Danesh-Meyer HV. The water-drinking test: the elegance of simplicity. Clin Experiment Ophthalmol. 2008;36(4):301-303.

72. Arora KS, Jefferys JL, Maul EA, Quigley HA. Choroidal thickness change after water drinking is greater in angle closure than in open angle eyes. Invest Ophthalmol Vis Sci. 2012 21;53(10):6393-6402.

73. Houle RE, Grant WM. Alcohol, vasopressin, and intraocular pressure. Invest Ophthalmol. 1967;6(2):145-154.

74. Mehra KS, Roy PN, Khare BB. Tobacco smoking and glaucoma. Ann Ophthalmol. 1976;8(4):462-464.

75. Järvinen T, Pate DW, Laine K. Cannabinoids in the treatment of glaucoma. Pharmacol Ther. 2002;95(2):203-220.

76. Porcella A, Maxia C, Gessa GL, Pani L. The synthetic cannabinoid WIN55212-2 decreases the intraocular pressure in human glaucoma resistant to conventional therapies. Eur J Neurosci. 2001;13(2): 409-412.

77. Beilin M, Neumann R, Belkin M, Green K, Bar-Ilan A. Pharmacology of the intraocular pressure (IOP) lowering effect of systemic dexanabinol (HU-211), a non-psychotropic cannabinoid. J Ocul Pharmacol Ther. 2000;16(3):217-230.

78. Zhan GL, Camras CB, Palmberg PF, Toris CB. Effects of marijuana on aqueous humor dynamics in a glaucoma patient. J Glaucoma. 2005;14(2):175-177.

79. Read SA, Collins MJ, Becker H, et al. Changes in intraocular pressure and ocular pulse amplitude with accommodation. Br J Ophthalmol. 2010;94(3):332-335.

80. Zeimer RC, Wilensky JT, Gieser DK, Mori MM, Baker JP. Evaluation of a self tonometer for home use. Arch Ophthalmol. 1983;101(11): 1791-1793.

81. Zeimer RC, Wilensky JT, Gieser DK, Welch DB, Mori MT, Kahanic D. Application of a self-tonometer to home tonometry. Arch Ophthalmol. 1986;104(1):49-53.

82. Fresco BB. A new tonometer - the pressure phosphene tonometer: clinical comparison with Goldman tonometry. Ophthalmology. 1998;105(11):2123-2126.

83. Alvarez TL, Gollance SA, Thomas GA, et al. The Proview phosphene tonometer fails to measure ocular pressure accurately in clinical practice. Ophthalmology. 2004;111(6):1077-1085.

84. Lam DS, Leung DY, Chiu TY, et al. Pressure phosphene self-tonometry: a comparison with goldmann tonometry in glaucoma patients. Invest Ophthalmol Vis Sci. 2004;45(9):3131-3136.

85. Chew GS, Sanderson GF, Molteno AC. The pressure phosphene tonometer - a clinical evaluation. Eye (Lond). 2005;19(6):683-685.

86. Li J, Herndon LW, Asrani SG, Stinnett S, Allingham RR. Clinical comparison of the Proview eye pressure monitor with the Goldmann applanation tonometer and the Tonopen. Arch Ophthalmol. 2004;122(8): $1117-1121$.

87. Danesh-Meyer HV, Niederer R, Gaskin BJ, Gamble G. Comparison of the Proview pressure phosphene tonometer performed by the patient and examiner with the Goldmann applanation tonometer. Clin Experiment Ophthalmol. 2004;32(1):29-32.

88. Gunvant P, Lievens CW, Newman JM 3rd, Gerstner MD, Chang F, Haine CL. Evaluation of some factors affecting the agreement between the Proview Eye Pressure Monitor and the Goldmann applanation tonometer measurements. Clin Exp Optom. 2007;90(4):290-295.

89. Kontiola A. A new electromechanical method for measuring intraocular pressure. Doc Ophthalmol. 1996-1997;93(3):265-276.

90. Kontiola A, Puska P. Measuring intraocular pressure with the Pulsair 3000 and Rebound tonometers in elderly patients without an anesthetic. Graefes Arch Clin Exp Ophthalmol. 2004;242(1):3-7.

91. Gandhi NG, Prakalapakorn SG, El-Dairi MA, Jones SK, Freedman SF. Icare ONE rebound versus Goldmann applanation tonometry in children with known or suspected glaucoma. Am J Ophthalmol. 2012;154(5): 843-849.
92. Flemmons MS, Hsiao YC, Dzau J, Asrani S, Jones S, Freedman SF. Home tonometry for management of pediatric glaucoma. Am J Ophthalmol. 2011;152(3):470-478.

93. Halkiadakis I, Stratos A, Stergiopoulos G, et al. Evaluation of the Icare-ONE rebound tonometer as a self-measuring intraocular pressure device in normal subjects. Graefes Arch Clin Exp Ophthalmol. 2012; 250(8):1207-1211.

94. Chui WS, Lam A, Chen D, Chiu R. The influence of corneal properties on rebound tonometry. Ophthalmology. 2008;115(1):80-84.

95. Asrani S, Zeimer R, Wilensky J, Gieser D, Vitale S, Lindenmuth $\mathrm{K}$. Large diurnal fluctuations in intraocular pressure are an independent risk factor in patients with glaucoma. J Glaucoma. 2000;9(2): 134-142.

96. Sit AJ. Continuous monitoring of intraocular pressure: rationale and progress toward a clinical device. J Glaucoma. 2009;18(4): 272-279.

97. Collins CC. Miniature passive pressure transensor for implanting in the eye. IEEE Trans Biomed Eng. 1967;14(2):74-83.

98. Walter P, Schnakenberg U, vom Bögel G, et al. Development of a completely encapsulated intraocular pressure sensor. Ophthalmic Res. 2000;32(6):278-284.

99. Walter P. Intraocular pressure sensor: where are we - where will we go? Graefes Arch Clin Exp Ophthalmol. 2002;240(5):335-336.

100. Chen PJ, Rodger DC, Saati S, Humayun MS, Tai Y-C. Microfabricated implantable parylene-based wireless passive intraocular pressure sensors. J Microelectromech Syst. 2008;17(6):1342-1351.

101. Downs JC, Burgoyne CF, Seigfreid WP, Reynaud JF, Strouthidis NG, Sallee V. 24-hour IOP telemetry in the nonhuman primate: implant system performance and initial characterization of IOP at multiple timescales. Invest Ophthalmol Vis Sci. 2011;52(10):7365-7375.

102. Greene ME, Gilman BG. Intraocular pressure measurement with instrumented contact lenses. Invest Ophthalmol. 1974;13(4): 299-302.

103. Cooper RL, Beale DG, Constable IJ. Passive radiotelemetry of intraocular pressure in vivo: calibration and validation of continual scleral guard-ring applanation transensors in the dog and rabbit. Invest Ophthalmol Vis Sci. 1979;18(9):930-938.

104. Cooper RL, Beale DG, Constable IJ, Grose GC. Continual monitoring of intraocular pressure: effect of central venous pressure, respiration, and eye movements on continual recordings of intraocular pressure in the rabbit, dog, and man. Br J Ophthalmol. 1979;63(12): 799-804.

105. Medeiros FA. Glaucoma risk factors: Fluctuations in intraocular pressure. In: Schacknow PN, Samples JN, editors. The Glaucoma Book: A Practical, Evidence-Based Approach to Patient Care. New York: Springer; 2010:51-54.

106. SENSIMED Triggerfish ${ }^{\circledR}-$ Continuous IOP Monitoring. Lausanne, Switzerland: Sensimed AG; 2013 [cited March 7, 2103]. Available from: http://www.sensimed.ch/en/products/sensimed-triggerfishr.html [webpage on the internet]. Accessed March 7, 2013.

107. Leonardi M, Leuenberger P, Bertrand D, Bertsch A, Renaud P. First steps toward noninvasive intraocular pressure monitoring with a sensing contact lens. Invest Ophthalmol Vis Sci. 2004;45(9):3113-3117.

108. Leonardi M, Pitchon EM, Bertsch A, Renaud P, Mermoud A. Wireless contact lens sensor for intraocular pressure monitoring: assessment on enucleated pig eyes. Acta Ophthalmol. 2009;87(4):433-437.

109. Hjortdal JO, Jensen PK. In vitro measurement of corneal strain, thickness, and curvature using digital image processing. Acta Ophthalmol Scand. 1995;73(1):5-11.

110. Lam AK, Douthwaite WA. The effect of an artificially elevated intraocular pressure on the central corneal curvature. Ophthalmic Physiol Opt. 1997;17(1):18-24.

111. Mansouri K, Shaarawy T. Continuous intraocular pressure monitoring with a wireless ocular telemetry sensor: initial clinical experience in patients with open angle glaucoma. Br J Ophthalmol. 2011;95(5): $627-629$. 
112. Mansouri K, Medeiros FA, Tafreshi A, Weinreb RN. Continuous 24-hour monitoring of intraocular pressure patterns with a contact lens sensor: safety, tolerability, and reproducibility in patients with glaucoma. Arch Ophthalmol. 2012;130(12):1534-1539.
113. Freiberg FJ, Lindell J, Thederan LA, Leippi S, Shen Y, Klink T. Corneal thickness after overnight wear of an intraocular pressure fluctuation contact lens sensor. Acta Ophthalmol. 2012;90(7): e534-e539.

\section{Clinical Optometry}

\section{Publish your work in this journal}

Clinical Optometry is an international, peer-reviewed, open access journa publishing original research, basic science, clinical and epidemiological studies, reviews and evaluations on clinical optometry. All aspects of patient care are addressed within the journal as well as the practice of optometry including economic and business analyses. Basic and clinical

Submit your manuscript here: http://www.dovepress.com/clinical-optometry-journal

\section{Dovepress}

research papers are published that cover all aspects of optics, refraction and its application to the theory and practice of optometry. The manuscript management system is completely online and includes a very quick and fair peer-review system, which is all easy to use. Visit http://www.dovepress. com/testimonials.php to read real quotes from published authors. 